Z Rheumatol 2012 $\cdot 71: 260$

DOI 10.1007/s00393-011-0912-5

(c) Springer-Verlag 2012
J. Wollenhaupt ${ }^{1} \cdot$ B. Swoboda ${ }^{2}$

${ }^{1}$ Klinikum Eilbek, Hamburg

${ }^{2}$ Orthopädisch-Rheumatologische Abteilung, FA Universität Erlangen-Nürnberg, Erlangen

\title{
Notfälle in der Rheumatologie
}

Auch wenn Rheumatologen nicht primär mit Notfallversorgung in Verbindung gebracht werden, gibt es einige wichtige Situationen, die dringliche Erkennung, rasche Diagnostik und zügige Therapie erfordern. Gerade die entzündlich rheumatischen Systemerkrankungen, aber auch die bakterielle Infektion von Gelenken können solche Notfälle darstellen.

Ziel des Themenschwerpunktes „Notfälle in der Rheumatologie" ist es, alle rheumatologisch tätigen Kolleginnen und Kollegen in Praxis und Klinik über die aktuelle Vorgehensweise bei ausgewählten Notfallsituationen unseres Fachgebietes zu informieren. Da die verzögerte Erkennung und Behandlung solcher kritischen Situationen den Behandlungserfolg wesentlich beeinflussen, sollten wir zügig das Richtige tun können.

\section{》) Die verzögerte}

Erkennung und Behandlung

kritischer Situationen

beeinflussen wesentlich

den Behandlungserfolg

Bei Kollagenosen und Vaskulitiden betreffen derartige Notfallsituationen die Lunge, die Niere und die Extremitätenperfusion. Experten der jeweiligen Krankheitskomplexe erläutern die Leitsymptome, den Weg zur Diagnosesicherung und die angemessenen therapeutischen Maßnahmen.

Während diese Situationen eher selten sind, stellt sich die Differenzialdiagnose "Gelenkinfektion" häufig, denn bei fast jeder mon- oder oligoartikulären Schubsituation einer Arthritis stellt die bakte- rielle Infektion eine Differenzialdiagnose dar. Deshalb versuchen wir, die praktische Vorgehensweise aus den interdisziplinären Erfahrungen von Rheumatologie und septischer Gelenkchirurgie gemeinsam zu betrachten.

Natürlich gibt es darüber hinaus auch andere Notfälle unseres Fachgebietes. Ihre ausführliche Darstellung hätte diesen Themenschwerpunkt gesprengt. Sie sollen daher durch andere Beiträge in späteren Heften der Zeitschrift für Rheumatologie dargestellt werden.

Ihre

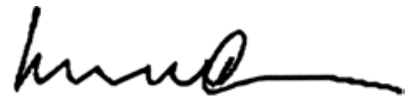

Jürgen Wollenhaupt

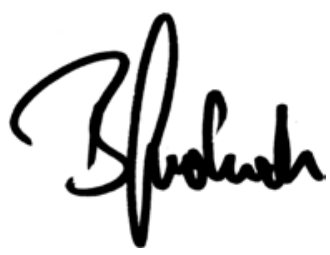

Bernd Swoboda

\section{Korrespondenzadressen}

Prof. Dr. J. Wollenhaupt

Klinikum Eilbek

Dehnhaide 120, 22081 Hamburg

jwollenhaupt@schoen-kliniken.de

Prof. Dr. B. Swoboda

Orthopädisch-Rheumatologische Abteilung,

FA Universität Erlangen-Nürnberg

Rathsberger Str. 57, 91054 Erlangen

bernd.swoboda@

ortho-rheuma.med.uni-erlangen.de 